\title{
Socialização em creche: um estudo sobre comportamento e brincadeiras de crianças pequenas
}

\author{
Marlene de Cássia Trivellato-Ferreira \\ Amanda Trivellato Ferreira \\ Centro Universitário Barão de Mauá, Ribeirão Preto - SP - Brasil \\ Edna Maria Marturano' \\ Faculdade de Medicina de Ribeirão Preto, USP, Ribeirão Preto - SP - Brasil
}

\begin{abstract}
Resumo: Objetivou-se comparar o engajamento das crianças com pares e nas atividades, antes e depois de participarem em brincadeiras coordenadas por um adulto na creche. Vinte crianças com idades entre 36 e 46 meses, em creche filantrópica, participaram de 20 sessões de brincadeiras. Precedendo e sucedendo esse período, elas foram filmadas por nove dias em atividades livres. Simultaneamente, uma observadora registrava comportamentos interativos e brincadeiras, focalizando cada criança por dois minutos. Esse registro era completado com a observação das filmagens. Categorizaram-se as brincadeiras em solitária, paralela, associativa e cooperativa, e os comportamentos como gregário, comunicação amigável com ou sem foco no brinquedo e comunicação hostil com ou sem foco no brinquedo. Os dados dos dois momentos foram comparados mediante uso do Teste de Wilcoxon. O aumento do tempo em brincadeira solitária e conjunta, o aumento das interações positivas e a diminuição das interações negativas sugerem maior engajamento após as atividades guiadas pela educadora.
\end{abstract}

Palavras-chave: creche; criança pré-escolar; recreação; engajamento; socialização.

\section{SOCIALIZATION IN DAY CARE CENTER: A STUDY ON CHILDREN BEHAVIOR AND PLAY}

\begin{abstract}
The objective was to compare the engagement of children with peers and in activities, before and after participating in teacher guided play in the day care center. Twenty children aged between 36 and 46 months, in a philanthropic day care center, participated in 20 play sessions. Preceding and succeeding that period, they were filmed during free play for nine days. Simultaneously, an observer recorded interactive and play behaviors, focusing on each child for two minutes, and later completed her recordings with the images captured by the cameras. Observed play was categorized as solitary, parallel, associative and cooperative. Interactive behaviors were categorized as gregarious, friendly communication focused or not on toy and unfriendly communication focused or not on toy. Data from the two observation blocks were compared by means of the Wilcoxon Test. Increased time in solitary and joint play, increased positive interactions and reduced negative interactions suggest greater engagement after teacher-guided play.
\end{abstract}

Keywords: day care, preschool child, recreation, engagement, socialization

1 Endereço para correspondência: Edna Maria Marturano, Faculdade de Medicina de Ribeirão Preto, Avenida Presidente Castelo Branco, 2450, Ribeirão Preto - SP - Brasil. CEP: 14096-560. E-mail: emmartur@ fmrp.usp.br. 
SOCIALIZACIÓN EN LA GUARDERÍA: LA CONDUCTA Y LOS JUEGOS DE NIÑOS PEQUEÑOS

\begin{abstract}
Resumen: El objetivo fue comparar la participación de niños con sus compañeros y actividades antes y después de participar en sesiones de juego coordinado por el adulto en la guardería. Veinte niños de edades comprendidas entre 36 y 46 meses en guardería sin fines de lucro participaron en 20 sesiones de juego. Anterior y posterior de esto, durante nueve días los niños fueron filmados y un observador registró sus comportamientos interactivos y juegos, centrándose en cada niño durante dos minutos. Los juegos se clasificaron en solitario, paralelo, asociativo y cooperativo. Los comportamientos se clasificaron como gregario, comunicación amable con o sin foco en el juguete y comunicación hostil con o sin foco en el juguete. Los datos de ambos momentos se compararon mediante la Prueba de Wilcoxon. El aumento del tiempo en el jugar solitario y juntos, el aumento de las interacciones positivas y la reducción de las interacciones negativas sugieren una mayor participación después de las actividades guiadas por el profesor.
\end{abstract}

Palabras clave: guardería; los niños de preescolar; recreación; participación; socialización.

A creche, ambiente coletivo em que a criança pequena tem a oportunidade de conviver cotidianamente com outras crianças fora do contexto da família, constitui um importante espaço de desenvolvimento da sociabilidade. Nesse contexto, tem sido observado que as crianças se envolvem em interações umas com as outras, já no primeiro ano de vida (Amorim, Anjos, \& Rossetti-Ferreira, 2012). Espera-se, assim, que a experiência em creche favoreça o engajamento, definido por McWilliam e Bailey (1992, apud Aguiar \& McWilliam, 2013) como o total de tempo que as crianças interagem com o ambiente, de forma apropriada ao contexto e ao seu nível de desenvolvimento e competência. O conceito de engajamento prediz que o desenvolvimento é promovido quando as crianças passam o tempo brincando ou participando de atividades, em vez de ficarem desocupadas ou emitindo comportamentos indesejáveis como chorar, bater nos colegas ou destruir materiais (Aguiar \& McWilliam, 2013).

No curso do desenvolvimento, há progressivo engajamento com o ambiente à medida que a criança cresce. Por exemplo, entre pré-escolares, ao passo que a agressão diminui com a idade (Côté, Vaillancourt, Barker, Nagin, \& Tremblay, 2007), a participação em brincadeiras com os pares é maior e mais sofisticada entre as crianças mais velhas (Parten, 1932).

Parten (1932) definiu categorias de participação com base na observação de brincadeiras espontâneas de crianças na creche. A categoria mais frequentemente observada foi a brincadeira paralela, em que a criança brinca perto de outras, com brinquedos semelhantes, mas não tenta influenciar ou modificar a atividade das demais. Dos 2 aos 4 anos, a brincadeira solitária e a paralela diminuem, ao passo que aumentam formas mais avançadas, como a brincadeira associativa e a cooperativa (Lordelo \& Carvalho, 2006; Parten, 1932).

Pesquisas recentes sugerem, porém, que a exposição precoce ao ambiente da creche em tempo integral pode contribuir para um menor engajamento. Morrissey (2010) constatou que o ingresso antes dos 3 anos de idade estava associado a desfechos de 
maior competência cognitiva, mas também a mais comportamentos externalizantes no final da educação infantil. No estudo de Coley, Votruba-Drzal, Miller e Koury (2013), a permanência em tempo integral, mas não em tempo parcial, em centros norte-americanos de Early Educational Care (EEC), quando iniciada antes dos 36 meses, foi associada posteriormente, na pré-escola, a menos comportamentos de atenção e persistência nas atividades, bem como mais comportamentos agressivos e disruptivos em relação aos pares. Em contrapartida, em escolas da Noruega, que têm nível homogêneo de qualidade alta, a maior exposição a cuidados educativos precoces, em termos de número de horas por semana, não foi associada a mais problemas externalizantes aos 18 e aos 36 meses (Zachrisson, Dearing, Lekhal, \& Toppelberg, 2013). Esses resultados podem ser vistos em uma perspectiva de qualidade do ambiente da creche, particularmente para crianças que se desenvolvem em condições adversas. Burchinal, Vandergrift, Pianta, \& Mashburn (2010) obtiveram indícios de que a qualidade do EEC é determinante para o engajamento comportamental, interpessoal e acadêmico em crianças de baixa renda; na metanálise conduzida por Keys et al. (2013), a qualidade do EEC foi fator de proteção para crianças cujas mães são menos escolarizadas, promovendo habilidades sociais nesse grupo.

A qualidade da creche como centro de cuidados educativos precoces pode ser incrementada por meio de programas interativos ajustados ao desenvolvimento da criança. Nesse rol estão programas como o High-Scope e o PATHS que, associados, promovem competência social (Moore et al., 2015) em crianças de 3 a 4 anos. Para a mesma faixa etária, também podem ser citadas atividades de iniciação artística e musical, que promovem autorregulação (Brown \& Sax, 2013), e a prática de narrativa e dramatização, que reduz os comportamentos agressivos nas brincadeiras e aumenta a autor regulação (Nicolopoulou, Cortina, Ilgaz, Cates, \& Sá, 2015).

Trabalhando com crianças mais jovens, de 21 a 26 meses, Pontoglio e Marturano (2010) inseriram no currículo de atividades da creche um programa de brincadeiras musicalizadas. O propósito do programa era promover o engajamento das crianças com os pares e também nas atividades que o espaço da creche oportuniza. Um pressuposto da intervenção foi que o engajamento pode ser favorecido com mediação apropriada na zona de desenvolvimento proximal (ZDP), definida por Vygotsky (2000, p. 112) como "a distância entre o nível de desenvolvimento real, que se costuma determinar através da solução independente de problemas, e o nível de desenvolvimento potencial, determinado através da solução de problemas sob a orientação de um adulto ou em colaboração com companheiros mais capazes". Com base nas proposições de Vygotsky (2000), as autoras supõem que as interações sociais e a brincadeira são instâncias em que as crianças funcionam na ZDP.

A intervenção foi ministrada a uma turma de 12 crianças, em 20 sessões, ao longo de dois meses. Os comportamentos das crianças foram registrados por um observador, antes e depois da intervenção, durante atividades livres. Por meio de comparações entre os dois momentos, constatou-se diminuição da brincadeira solitária, aumento 
da brincadeira paralela e tendência ao aumento da brincadeira associativa. Ao mesmo tempo, as interações amigáveis entre as crianças aumentaram. Os resultados sugerem que, no curto período de dois meses, as crianças expostas ao programa ampliaram e sofisticaram seu engajamento no ambiente.

Nesse contexto se inscreve o presente estudo, em que se acompanhou uma turma de crianças de baixa renda, com idades de 3 a 4 anos e história de ingresso precoce em creche, antes dos 36 meses. Seu objetivo foi comparar o engajamento das crianças com pares e nas atividades, antes e depois de participarem do programa proposto por Pontoglio e Marturano (2010). Esperava-se elevação no engajamento, expressa em aumento na participação em brincadeiras evolutivamente mais elaboradas (Lordelo \& Carvalho, 2006), aumento nas interações com os pares (Eggum-Wilkens et al., 2014) e redução nos comportamentos disruptivos, como agressão e disputas (Côté et al., 2007).

\section{Método}

\section{Participantes}

Participaram do estudo 20 crianças (13 meninos) da turma do Maternal II de uma creche filantrópica em um município do interior paulista. As crianças permaneciam na creche de segunda a sexta-feira, das $7 \mathrm{~h} 30$ às 16h30. As atividades diárias da turma eram conduzidas por duas educadoras. A idade dos participantes variava entre 36 e 46 meses (média 40 meses) no início da pesquisa. Todos tinham frequentado creche por pelo menos um ano. Doze provinham da mesma turma do Maternal I no ano anterior. Oito eram recém-transferidos de outras creches. As famílias tinham renda per capita não superior a um e meio salário mínimo.

\section{Instrumentos e materiais}

Foram utilizadas duas filmadoras de alta definição Sony SR20 para o registro das sessões de atividade livre das crianças. As câmeras foram posicionadas na sala onde as atividades foram realizadas, em ângulos opostos, de modo a possibilitar uma captação panorâmica das imagens. Folhas A4 e lápis foram usados para o registro das observações. Também se usaram dois conjuntos de brinquedos novos, um em cada período de observação.

\section{Procedimentos}

Considerações éticas. A pesquisa atende às diretrizes e normas da Resolução 466/12 do Conselho Nacional de Saúde do Ministério da Saúde e ao disposto na Resolução $n^{\circ}$ 016/2000 do Conselho Federal de Psicologia. O projeto foi aprovado pelo Comitê de Ética em Pesquisa do Centro Universitário Barão de Mauá (Processo n 594/2011). A educadora que aplicava o programa tinha experiência na sua condução e era familiar aos participantes. Durante a intervenção, esteve presente pelo menos uma das educadoras responsáveis pela turma, disponível para dar assistência às crianças em necessi- 
dades emergentes. Os pais ou responsáveis assinaram termo de consentimento livre e esclarecido para a participação de seus filhos e gravação das imagens para fins exclusivos da pesquisa. A eles foi assegurado o acesso às informações referentes a seus fiIhos, a qualquer momento, durante a pesquisa.

\section{Coleta de dados}

Filmagens foram tomadas durante períodos de atividade livre que fazem parte da rotina diária da instituição. Dois procedimentos foram combinados. A par com o registro contínuo pelas filmadoras fixas, a primeira autora, observadora treinada, familiar às crianças, registrou os comportamentos dos participantes em rodízio, por períodos de dois minutos. Imediatamente depois de concluída cada sessão, a observadora assistia às filmagens, completando seu registro manual. Tal combinação atendeu a dois requisitos de confiabilidade dos dados: a observadora presente na sala pôde registrar as verbalizações da criança focal e de seus interlocutores, o que não seria possível só com as câmeras, devido ao ruído circundante no espaço coletivo; e as câmeras permitiram preservar as imagens para reconstituição da sequência de eventos, o que não seria exequível só com o registro manual.

Em cada sessão de observação, o registro manual e a filmagem eram feitos até que se completasse o rodízio de observação de todos os participantes. A ordem em que as crianças seriam observadas em uma sessão era estabelecida previamente por sorteio.

\section{Observação pré-intervenção}

As observações ocorreram diariamente nas duas semanas iniciais de atividades da turma, antecedendo o início do programa. As atividades livres durante as quais as crianças foram observadas ocorriam sempre na mesma sala, com a presença das duas educadoras da turma e da observadora. A sala era suprida com brinquedos de faz de conta (animais, bonecas, ferramentas, carrinhos, telefones, utensílios domésticos etc.), blocos de construção e quebra-cabeças. Havia quantidade suficiente deles, porém não mais que quatro ou cinco brinquedos iguais. Os brinquedos eram dispostos no chão, para acesso livre das crianças. Foram completadas 10 a 11 sessões de observação por participante.

\section{Intervenção}

A intervenção compreende atividades lúdicas realizadas coletivamente. Em uma sessão típica, as crianças e as educadoras se sentam no chão em círculo; um tema de interesse (por exemplo, animais, folclore, nosso corpo) é introduzido por meio de uma breve exposição ou história dialogada, seguida de atividade com música (por exemplo, "Sapo Cururu", "Tindolelê"), em que se explora movimento, ritmo, expressão corporal, linguagem oral e gestual, imitação direta e diferida, seguimento de instruções. Segue-se uma brincadeira que demanda interações entre as crianças, como revezamento, dar 
e receber, permutar, dar e seguir o modelo de ação. As atividades podem ser mediadas por objetos comercializados ou manufaturados como máscaras, bichos de pelúcia, bolas de borracha, dobraduras de origami ou instrumentos de percussão. No período de realização da pesquisa, as crianças participaram de 20 sessões, com duração de 15 a 30 minutos, duas vezes por semana. No tempo restante de permanência na creche, participavam da programação regular: roda de conversa, contação de história, projetos, atividade livre com brinquedos, recreação no parquinho, rotinas de alimentação e higiene, cinema, celebração de datas previstas no calendário escolar.

\section{Observação pós-intervenção}

Nas semanas que sucederam a $20^{\mathrm{a}}$ sessão da intervenção, as crianças foram observadas nas mesmas condições da observação pré-intervenção. Diversas crianças faltaram, o que demandou prolongamento das observações para três semanas. Mesmo assim, algumas crianças terminaram o ciclo com nove observações. Encerrou-se o procedimento na terceira semana, mesmo sem ter sido atingida a meta de 10 observações por criança, para não aumentar a diferença de tempo entre as duas fases de coleta de dados.

\section{Análise dos registros das sessões}

Logo em seguida ao término de cada sessão de observação ao vivo, a observadora completava seus registros com a transcrição das filmagens, utilizando a técnica de observação por sujeito focal (Altmann, 1974). Nessa tarefa, era necessário localizar cada criança no trecho de gravação das imagens correspondentes aos dois minutos em que a observadora havia focalizado aquela mesma criança no rodízio. Estabelecida a correspondência entre o início do trecho de gravação e o início do registro da observação ao vivo, a transcrição era feita em duas janelas de um minuto. A cada minuto se fazia pausa na reprodução da gravação para descrição cursiva dos comportamentos da criança, em linguagem que atendesse aos requisitos de objetividade, precisão e clareza. As pessoas com quem a criança manteve contato no intervalo foram identificadas.

\section{Análise dos dados}

Para cada participante, foram selecionados os registros de nove sessões de observação da primeira fase, para possibilitar a comparação entre fases. O critério para essa seleção foi descartar a primeira e, se necessário, a última observação da fase pré-intervenção. Foram feitas duas leituras da transcrição das sessões de atividade livre, uma com foco nas brincadeiras e a outra com foco nos comportamentos orientados para os pares.

A participação em brincadeiras foi classificada, de acordo com as classes propostas por Parten (1932), em: Ausência de brincadeira - a criança não parece estar brincando (inclui as categorias desocupado e observador de Parten); Brincadeira solitária - a criança brinca sozinha, seja com algum objeto, seja com seu próprio corpo, e não estabelece nenhuma interação social relacionada ao conteúdo e pro- 
pósito da brincadeira, nem faz qualquer esforço para se aproximar de outras crianças; Brincadeira paralela - a criança brinca próxima a um ou mais companheiros, com brinquedos semelhantes ou sobre um mesmo tema, porém de maneira independente e não necessariamente igual, sem interagir quanto à brincadeira e sem tentar influenciar o brincar do outro; Brincadeira associativa - duas ou mais crianças brincam juntas, de modo semelhante, sobre o mesmo tema; elas interagem, seguem umas às outras, emprestam e tomam emprestados brinquedos, porém não há divisão de tarefas e nenhuma organização; Brincadeira cooperativa - há, na brincadeira de duas ou mais crianças sobre o mesmo tema, cooperação ou competição, divisão de tarefas e de papéis.

Para análise dos comportamentos orientados para os pares, foi construído um sistema de categorias com base nos indicadores propostos por Howes \& Matheson (1992) em seu estudo sobre brincadeiras de pré-escolares em centros coletivos de cuidados diários. Os comportamentos observados foram classificados e agrupados em seis categorias amplas: Sozinho - comportamentos que não envolvem contato com outras pessoas, inativo nas classes, observa colegas, voltado para o próprio corpo, ocupado com brinquedo; Gregário - contatos amistosos entre as crianças, não envolvendo brinquedos, nas classes inicia contato, é alvo de iniciativa de contato, responde positivamente à iniciativa do colega, interage verbalmente; Isolado - tentativas frustradas de contato, nas classes inicia contato sem resposta, ignora a iniciativa do colega; Comunicação amigável com foco no brinquedo - contatos amistosos entre as crianças, sempre envolvendo brinquedos, como solicita brinquedo, pede para brincar junto, convida para brincar, negocia, coopera, compartilha, participa; Comunicação não amigável com foco no brinquedo - contatos inamistosos entre as crianças, sempre envolvendo brinquedos, como toma o brinquedo, disputa o brinquedo, recusa a cooperar, recusa a compartilhar; Comunicação hostil - contatos não amistosos entre as crianças, não envolvendo brinquedos, nas classes agride fisicamente, agride verbalmente, rejeita o colega, é agredido fisicamente, é agredido verbalmente, é rejeitado pelo colega.

Utilizando-se o coeficiente de correlação de Spearman, o índice médio de concordância entre dois juízes, ao utilizarem o sistema na classificação dos comportamentos de cinco crianças, foi 0,79, variando entre 0,98 e 0,57. Correlações mais altas ocorreram para as classes de comportamentos mais frequentes e as mais baixas, para as menos frequentes.

A fim de investigar possíveis diferenças entre os dois momentos de observação, a frequência de cada classe de brincadeira e de comportamento foi computada, por período de observação, para cada criança. Também foi estimado o tempo gasto em atividade ininterrupta na brincadeira, com base no número de intervalos de dois minutos em que se observou a criança envolvida somente em uma classe de brincadeira. Os dados assim processados nos dois períodos foram transpostos para planilhas do programa SPSS v. 21 e comparados por meio do Teste de Wilcoxon para amostras dependentes. O nível de significância foi $p \leq 0,05$. 
Considerando que a familiaridade entre as crianças poderia influenciar os resultados, o teste $U$ de Mann Whitney foi usado para comparar os dados do grupo dos novatos na creche $(n=8)$ com os do grupo proveniente do Maternal I $(n=12)$, antes e depois da intervenção.

\section{Resultados}

Na Tabela 1 estão as médias, medianas e valores mínimo e máximo das classes de parceria na brincadeira, antes e depois da intervenção, bem como os valores de $Z$ e $p$ obtidos com o Teste de Wilcoxon. Observa-se diminuição na frequência das categorias Ausência de brincadeira e Brincadeira solitária, assim como aumento na categoria Brincadeira paralela.

\section{Tabela I. Estatísticas descritivas referentes às classes de parceria social na brincadeira, antes e depois da intervenção}

\begin{tabular}{|c|c|c|c|c|c|c|c|c|c|c|}
\hline \multirow[b]{2}{*}{$\begin{array}{l}\text { Classe de parceria na } \\
\text { brincadeira }\end{array}$} & \multicolumn{4}{|c|}{ Antes da intervenção } & \multicolumn{6}{|c|}{ Depois da intervenção } \\
\hline & M & $M d n$ & Mínimo & Máximo & M & Mdn & Mínimo & Máximo & z & p \\
\hline Ausência de brincadeira & 1,55 & 1,00 & 0 & 5 & 0,60 & 0,00 & 0 & 4 & 2,048 & $0,041^{*}$ \\
\hline Brincadeira solitária & 6,85 & 7,00 & 2 & II & 4,80 & 5,00 & I & 8 & 3,010 & $0,003 *$ \\
\hline Brincadeira paralela & 1,40 & 2,00 & 0 & 4 & 2,35 & 2,00 & 0 & 5 & 2,342 & $0,019 *$ \\
\hline Brincadeira associativa & 2,10 & 2,50 & 0 & 4 & 2,60 & 2,50 & 0 & 5 & 1,204 & 0,229 \\
\hline Brincadeira cooperativa & 0,00 & 0,00 & 0 & 0 & 0,10 & 0,00 & 0 & I & $|, 4| 4$ & 0,157 \\
\hline
\end{tabular}

Nota: $\mathrm{N}=20$. Mdn: mediana. Teste de Wilcoxon para amostras dependentes. ${ }^{*} p<0,05$.

Fonte: Elaborada pelas autoras.

Na Tabela 2 se encontram os resultados da estimativa do tempo que as crianças despenderam em cada categoria de brincadeira. Com um tempo máximo possível de 18 minutos em cada período, produto de nove observações de dois minutos, observa-se que as crianças se envolveram a maior parte do tempo em Brincadeira solitária, com aumento significativo depois da intervenção. Assim, houve ao mesmo tempo redução da frequência (Tabela 1) e aumento da duração (Tabela 2) dos episódios de Brincadeira solitária. Também aumentou o tempo ocupado em Brincadeira conjunta, quando se considera a soma das categorias Brincadeira associativa e Brincadeira cooperativa. Uma tendência de aumento $(p=0,057)$ foi observada no tempo despendido em Brincadeira paralela. 
Tabela 2. Tempo gasto em atividade ininterrupta na brincadeira, antes e depois da intervenção'

\begin{tabular}{lllllllllllll}
\hline & \multicolumn{1}{c}{ Antes da intervenção } & \multicolumn{7}{c}{ Depois da intervenção } \\
$\begin{array}{l}\text { Classe de parceria } \\
\text { na brincadeira }\end{array}$ & Média & Mdn & Mínimo & Máximo & Média & Mdn & Mínimo & Máximo & Z & P \\
\hline Ausência de brincadeira & 0,50 & 0,00 & 0 & 3 & 0,25 & 0,00 & 0 & 2 & 0,997 & 0,319 \\
Brincadeira solitária & 4,00 & 4,00 & 0 & 12 & 7,00 & 7,00 & 0 & 12 & 2,765 & $0,006 *$ \\
Brincadeira paralela & 0,70 & 0,00 & 0 & 4 & 1,90 & 2,00 & 0 & 6 & 1,904 & 0,057 \\
Brincadeira associativa & 1,50 & 2,00 & 0 & 4 & 2,90 & 2,00 & 0 & 8 & 1,882 & 0,060 \\
Brincadeira cooperativa & 0,00 & 0,00 & 0 & 0 & 0,20 & 0,00 & 0 & 2 & 1,414 & 0,157 \\
Brincadeira conjunta & 1,50 & 2,00 & 0 & 4 & 3,10 & 2,00 & 0 & 8 & 2,026 & $0,043 *$ \\
(associativa + cooperativa) & & & & & & & & & & & & \\
\hline
\end{tabular}

Nota: $\mathrm{N}=20$. Mdn: mediana. Teste de Wilcoxon para amostras dependentes. ${ }^{*} p<0,05$.

'Estimativa em minutos, obtida sobre o número de intervalos de dois minutos em que se observou a criança envolvida somente em uma classe de brincadeira.

Fonte: Elaborada pelas autoras.

Nos resultados referentes às classes de comportamento orientado para os colegas, apresentados na Tabela 3, houve tendência geral de redução das médias, com exceção da categoria Gregário, cuja frequência aumentou significativamente depois da intervenção. Redução significativa ocorreu nas categorias Sozinho e Comunicação Hostil.

\section{Tabela 3. Estatísticas descritivas referentes às classes de comportamento orientado para pessoas, antes e depois da intervenção}

\begin{tabular}{llllllllllll}
\hline & \multicolumn{1}{c}{ Antes da intervenção } & \multicolumn{1}{c}{ Depois da intervenção } \\
Classe de comportamento & Média & Mdn & Mínimo & Máximo & Média & Mdn & Mínimo & Máximo & Z & $\mathrm{p}$ \\
\hline Sozinho & 16,50 & 16,00 & 4,00 & 24,00 & 11,60 & 12,00 & 2,00 & 18,00 & 2,795 & $0,005^{*}$ \\
Isolado & 0,70 & 0,50 & 0,00 & 2,00 & 0,50 & 0,00 & 0,00 & 2,00 & 0,884 & 0,377 \\
Gregário & 2,90 & 2,50 & 0,00 & 9,00 & 4,55 & 4,00 & 0,00 & 16,00 & 2,057 & $0,040 *$ \\
$\begin{array}{l}\text { Comunicação amigável } \\
\text { com foco no brinquedo }\end{array}$ & 4,35 & 4,50 & 0,00 & 11,00 & 2,95 & 2,00 & 0,00 & 8,00 & 1,832 & 0,067 \\
$\begin{array}{l}\text { Comunicação não amigável } \\
\text { com foco no brinquedo }\end{array}$ & 2,35 & 2,00 & 0,00 & 9,00 & 1,15 & 0,00 & 0,00 & 5,00 & 1,645 & 0,100 \\
\begin{tabular}{l} 
Comunicação hostil \\
\hline
\end{tabular} & 1,55 & 1,00 & 0,00 & 6,00 & 0,35 & 0,00 & 0,00 & 1,00 & 2,327 & $0,020 *$ \\
\hline
\end{tabular}

Nota: $\mathrm{N}=20$. Mdn: mediana. Teste de Wilcoxon para amostras dependentes. ${ }^{*} p<0,05$.

Fonte: Elaborada pelas autoras.

Foi feita uma análise adicional das interações, somando-se, entre os comportamentos orientados para os colegas, as frequências daqueles com conotação positiva (Gregário, Comunicação amigável com foco no brinquedo) em contraposição aos de conotação negativa (Comunicação não amigável com foco no brinquedo, Comunica- 
ção hostil) e segregando-se, em cada um desses dois agrupamentos, as situações em que a criança iniciava o contato ou era alvo da iniciativa de um colega. Também foi feita a comparação entre os dois momentos, com relação ao total de comportamentos orientados para os colegas. Os resultados dessas duas análises são apresentados no próximo parágrafo.

Os resultados do Teste de Wilcoxon indicaram aumento nas interações positivas que a criança observada iniciou $(Z=2,219, p=0,026)$, redução nas suas iniciativas de interação com conotação negativa $(Z=3,198, p=0,001)$ e redução nas interações negativas de que ela foi alvo $(Z=2,810, p=0,005)$. As interações positivas de que a criança foi alvo tiveram aumento, porém não significativo $(Z=1,691, p=0,091)$. Não houve diferença entre os dois momentos no total de interações, quer positivas ou negativas $(Z=0,764, p=0,445)$.

Nas comparações entre o grupo dos novatos e o dos egressos do Maternal I em todas as variáveis, apenas uma diferença foi encontrada. Os egressos do Maternal I apresentaram, antes da intervenção, mais comportamentos com conotação negativa $(Z=2,297, p=0,022)$

\section{Discussão}

Neste estudo, foi feito o acompanhamento de crianças com idades entre 36 e 46 meses, que compunham a turma do Maternal II em uma creche. Todas as crianças provinham de famílias de baixa renda e tinham experiência prévia de pelo menos um ano em creche de tempo integral. As crianças foram observadas em atividade livre com brinquedos, no início do funcionamento da turma e após dois meses, período em que participaram de um programa de brincadeiras musicalizadas conduzido por uma educadora. No pressuposto de que tais atividades seriam facilitadoras do engajamento das crianças no ambiente, esperava-se aumento na participação em brincadeiras evolutivamente mais elaboradas e nas interações com os pares, bem como redução nos comportamentos disruptivos, como agressão e disputas.

Por meio de duas leituras dos registros das observações e filmagens, contemplando, respectivamente, a participação da criança nas brincadeiras e seus comportamentos em relação aos colegas, foi possível observar mudanças sugestivas de aumento do engajamento da criança em atividades e interações no ambiente natural (Aguiar \& McWilliam, 2013). Com efeito, a participação em brincadeiras evolutivamente mais elaboradas aumentou e os comportamentos com conotação negativa diminuíram. Em contrapartida, não se observou aumento na ocorrência de comportamentos orientados para os colegas.

Algumas mudanças observadas na participação em brincadeiras correspondem, em linhas gerais, ao padrão previamente observado em crianças de 2 a 4 anos em contexto de creche: no curto período de dois meses a partir do início das atividades da turma, aumentou o tempo que as crianças ocupavam em brincadeira conjunta, envolvendo as modalidades associativa e cooperativa, sugerindo aumento na participação social 
(Lordelo \& Carvalho, 2006; Parten, 1932; Pontoglio \& Marturano, 2010). Em contrapartida, também aumentou o tempo em brincadeira solitária, um resultado que à primeira vista contradiz os achados empíricos de Parten (1932) e Lordelo \& Carvalho (2006). Mas a contradição pode ser apenas aparente. Com efeito, no presente estudo, ao mesmo tempo que aumentou o tempo médio em brincadeira solitária, diminuiu sua frequência; tendência semelhante, em termos de frequência, foi observada por Lordelo \& Carvalho (2006), que constataram menor frequência de brincadeira individual entre as crianças mais velhas.

Os resultados relativos à brincadeira solitária merecem atenção. A situação de observação envolveu, nos dois momentos de coleta de dados, o acesso a brinquedos novos, que as crianças ainda não tinham tido a oportunidade de explorar. Nesse cenário, o aumento do tempo em brincadeira solitária, associado à redução de frequência dessa mesma classe e das classes Ausência de brincadeira (Tabela 1) e Sozinho (Tabela 3), denota um padrão de mudanças interessante. No início, as crianças interrompiam mais frequentemente sua atividade, alternando períodos curtos (menores que dois minutos) em que ora se ocupavam com algum brinquedo, ora observavam a sala ou simplesmente nada faziam. Depois de dois meses, elas passaram a se concentrar mais na exploração do brinquedo, o que pode ser considerado uma mudança positiva, no sentido de que se tornaram mais capazes de manter a atenção em uma atividade interessante, resistindo, mais que antes, aos diversos estímulos presentes na situação (movimentação dos colegas, outros brinquedos etc.). À luz do conceito de engajamento, as mudanças são sugestivas de progresso no nível de engajamento, denotando aumento da atenção e da persistência na atividade (Aguiar \& McWilliam, 2013).

Tendência semelhante pôde ser constatada nos comportamentos em relação aos colegas, ainda que não se observasse aumento no total de comportamentos orientados aos pares (Eggum-Wilkens et al., 2014): diminuíram as interações com conotação negativa, que antes da intervenção envolviam mais as crianças familiarizadas com a creche, e aumentaram as interações com conotação positiva. Assim, o aumento do engajamento com o ambiente, observado em relação às brincadeiras, ocorreu também no relacionamento entre as crianças. Esse é um resultado relevante, pois todas as crianças participantes do estudo frequentavam creche em tempo integral há pelo menos um ano. Conforme assinalado na introdução, alguns autores reportam efeitos adversos da exposição precoce a centros de cuidados educativos coletivos em tempo integral, afetando a sociabilidade (Coley et al., 2013; Morrissey, 2010).

Em conjunto, os resultados são compatíveis com desfechos observados em creches de qualidade, no que diz respeito ao engajamento comportamental e interpessoal (Burchinal et al., 2010; Keys et al., 2013). Eles reproduzem achados de investigações que avaliaram efeitos de intervenções voltadas à promoção do desenvolvimento de crianças pequenas em espaços coletivos: aumento de comportamentos pró-sociais (Moore et al., 2015), diminuição de comportamentos agressivos (Nicolopoulou et al., 
2015) e aumento do tempo de engajamento na brincadeira, possivelmente refletindo maior autorregulação (Brown \& Sax, 2013).

A respeito das habilidades de autorregulação, Brown \& Sax (2013) lembram que essas podem ser facilitadas pela exposição da criança a experiências de música e movimento como começar-parar, lento-rápido e forte-fraco. Essas modalidades de experiência foram promovidas nas sessões do programa conduzido com as crianças do presente estudo, associadas ao treinamento em habilidadess sociais, tais como esperar sua vez e revezar. Tal combinação pode ter contribuído para que, após 20 sessões, as interações amigáveis tenham aumentado e as interações hostis diminuído. Pode-se supor que o educador, mediando as interações nas brincadeiras guiadas, operou na zona de desenvolvimento proximal (Vygotsky, 2000), favorecendo a autorregulação e as interações sociais positivas.

No entanto, na ausência de um grupo de comparação não exposto ao programa, não se pode concluir que as mudanças positivas no nível e na qualidade do engajamento dos participantes sejam devidas à intervenção. Seria necessário efetivar investigação com delineamento de comparação entre um grupo exposto e o outro não exposto ao programa para verificar se as mudanças positivas identificadas no comportamento das crianças podem ser atribuídas à intervenção. Além disso, é preciso considerar que a presente investigação revelou mudanças ao longo de apenas dez semanas, havendo necessidade de estudos de seguimento para verificar sua permanência no tempo. Uma terceira limitação é o foco em uma só turma de uma única creche, o que restringe a generalização dos resultados.

Mesmo com as limitações apontadas, a pesquisa contribui para o estudo do desenvolvimento social das crianças, com apoio em um método de observação que se mostrou sensível para detectar mudanças, em curto período de tempo, compatíveis com previsões derivadas do conceito de engajamento. Seus resultados contribuem também para o campo aplicado da educação infantil, na medida em que apresentam indícios de efetividade de um programa de atividades lúdicas musicalizadas para promover a socialização das crianças e seu engajamento com o ambiente, em contexto de creche.

\section{Referências}

Aguiar, C., \& McWilliam, R. A, (2013). Consistency of toddler engagement across two settings. Early Childhood Research Quarterly, 28(1), 102- 110. DOI: 10.1016/j. ecresq.2012.04.003.

Altmann, J. (1974). Observational study of behavior: sampling methods. Behavior, 49(3), 227-265. DOI: 10.1163/156853974X00534.

Amorim, K. S., Anjos, A. M., \& Rossetti-Ferreira, M. C. (2012). Processos interativos de bebês em creche. Psicologia: Reflexão e Crítica, 25(2), 378-389. DOI: 10.1590/ S0102-79722012000200020. 
Brown, E., \& Sax, K. L. (2013). Arts enrichment and preschool emotions for lowincome children at risk. Early Childhood Research Quarterly, 28(2), 337- 346. DOI: 10.1016/j.ecresq.2012.08.002.

Burchinal, M., Vandergrift, N., Pianta, R., \& Mashburn, A. (2010). Threshold analysis of association between child care quality and child outcomes for low-income children in pre-kindergarten programs. Early Childhood Research Quarterly, 25(2), 166-176. DOI: 10.1016/j.ecresq.2009.10.004.

Coley, R. L., Votruba-Drzal, E., Miller, P. L., \& Koury, A. (2013). Timing, extent, and type of child care and children's behavioral functioning in kindergarten. Developmental Psychology, 49(10), 1859-1873. DOI: 10.1037/a0031251.

Côté, S. M., Vaillancourt, T., Barker, E. D., Nagin, D., \& Tremblay, R. E. (2007). The joint development of physical and indirect aggression: predictors of continuity and change during childhood. Development and Psychopathology, 19(1), 37-55. DOI: $10.10170 /$ S0954579407070034.

Eggum-Wilkens, N. D.; Fabes, R. A.; Castle, S.; Zhang, L.; Hanish, L. D., \& Martin, C. L. (2014). Playing with others: head Start children's peer play and relations with kindergarten school competence. Early Childhood Research Quarterly, 29, 345356. Doi 10.1016/j.ecresq.2014.04.008

Howes, C., \& Matheson, C. C. (1992). Sequences in the development of competent play with peers: social and social pretend play. Developmental Psychology, 28(5), 961-974. DOI: 10.1037/0012-1649.28.5.961.

Keys, T. D., Farkas, G., Burchinal, M. R., Duncan, G. J., Vandell, D. L., Li, W., Ruzek, E. A, \& Howes, C. (2013). Preschool center quality and school readiness: quality effects and variation by demographic and child characteristics. Child Development, 84(4), 1171-1190. DOI: 10.1111/cdev.12048.

Lordelo, E. R., \& Carvalho, A. M. A. (2006). Padrões de parceria social e brincadeira em ambiente de creches. Psicologia em Estudo, 11(1), 99-108. DOI: 10.1590/ S1413-73722006000100012.

Moore, J. E., Cooper, B. R., Domitrovich, C. E., Morgan, N. R., Cleveland, M. J., Shah, H., Jacobson, L., \& Greenberg, M. T. (2015). The effects of exposure to an enhanced preschool program on the social-emotional functioning of at-risk children. Early Childhood Research Quarterly, 32, 127-138. DOI: 10.1016/j. ecresq.2015.03.004.

Morrissey, T. W. (2010). Sequence of child care type and child development: what role does peer exposure play? Early Childhood Research Quarterly, 25(1), 33-50. DOI: 10.1016/j.ecresq.2009.08.005.

Nicolopoulou, A., Cortina, K. S., Ilgaz, H., Cates, C. B., \& Sá, A. B. de (2015). Using a narrative-and play-based activity to promote low-income preschoolers' oral 
language, emergent literacy, and social competence. Early Childhood Research Quarterly, 31, 147-162. DOI: 10.1016/j.ecresq.2015.01.006.

Parten, M. (1932). Social participation among preschool children. Journal of $A b-$ normal and Social Psychology, 27(3), 243-269.

Pontoglio, C. F., \& Marturano, E. M. (2010). Brincando na creche: atividades com crianças pequenas. Estudos de Psicologia (Campinas), 27(3), 365-373. DOI: 1590/ S0103-166X2010000300008.

Vygotsky, L. S. (2000). A formação social da mente (6a ed.). São Paulo: Martins Fontes.

Zachrisson, H. D., Dearing, E., Lekhal, R., \& Toppelberg, C. O. (2013). Little evidence that time in child care causes externalizing problems during early childhood in Norway. Child Development, 84(4), 1152-1170. DOI: 10.1111/cdev.12040.

Submissão: 10.12.2015

Aceitação: 14.9.2016 